\title{
Validación del test para la identificación de trastornos por uso de alcohol en población universitaria: AUDIT y AUDIT-C
}

\section{Validation of the Alcohol Use Disorders Identification Test in university students: AUDIT and AUDIT-C}

\author{
Miguel Ángel García Carretero*, José Pedro Novalbos Ruiz **, José Manuel Martínez \\ Delgado ***, Cristina O’Ferrall González **** \\ * Grupo PAIDI CTS-391 Grupo Multidisciplinario para el progreso de la Salud Mental, Universidad de Cádiz; ** Facultad de \\ Medicina, Universidad de Cádiz; *** Centro de Tratamiento de Adicciones. Diputación Provincial de Cádiz; **** Facultad de \\ Enfermería y Fisioterapia, Universidad de Cádiz.
}

\section{Resumen}

El objetivo de este trabajo fue determinar las propiedades psicométricas de los cuestionarios Alcohol Use Disorders Identification Test (AUDIT y AUDIT-C) para la detección de problemas por consumo de alcohol en universitarios. Participaron 1309 estudiantes, utilizando el Diario de consumo semanal como patrón de referencia y para el análisis de fiabilidad y validez el alfa de Cronbach, análisis exploratorio, índice kappa, coeficiente de correlación de Spearman y curvas ROC para los diferentes puntos de corte. Un 3,9\% de hombres y un $4 \%$ de mujeres con consumo de riesgo bajo presentaba consumo intensivo de alcohol (BD). AUDIT identificó un 20,1\% de Bebedor de riesgo y un 6,4\% de Bebedor con problemas físico-psíquicos y probable dependencia alcohólica. El instrumento presentó un alfa-Cronbach de 0,75 demostrando buena consistencia interna. Los puntos de corte óptimos fueron ocho para Bebedor de riesgo en hombres y seis para mujeres; trece puntos para Bebedor con problemas físico-psíquicos y probable dependencia alcohólica en ambos sexos; con AUDIT-C, fueron para Consumo de riesgo cinco para hombres y cuatro para mujeres. La validez de criterio para detectar consumo intensivo de alcohol (BD) con ambas versiones presentó un valor K moderado. Los resultados indican que AUDIT cuenta con adecuadas propiedades psicométricas para detectar precozmente problemas de consumo de alcohol en universitarios, recomendándose reducir su punto de corte a ocho en varones. El AUDIT-C mejora su poder predictivo aumentando en una unidad el punto de corte. Se recomienda revisar los ítems 2 y 3 para aumentar el valor predictivo del BD.

Palabras clave: Trastornos relacionados con el alcohol, Psicometría, Universitarios, AUDIT, AUDIT-C.

\begin{abstract}
The aim of this study was to determine the psychometric properties of the Alcohol Use Disorders Identification Test (AUDIT and AUDIT-C) in order to detect problems related to the consumption of alcohol in the university population. The sample consisted of 1309 students. A Weekly Alcohol Consumption Diary was used as a gold standard; Cronbach's Alpha, the Kappa index, Spearman's correlation coefficient and exploratory factor analysis were applied for diagnostic reliability and validity, with ROC curves used to establish the different cut-off points. Binge Drinking (BD) episodes were found in $3.9 \%$ of men and $4.0 \%$ of women with otherwise low-risk drinking patterns. AUDIT identified $20.1 \%$ as high-risk drinkers and $6.4 \%$ as drinkers with physical-psychological problems and probable alcohol dependence. Cronbach's alpha of 0.75 demonstrates good internal consistency. The best cut-off points for high-risk drinking students were 8 for males and 6 for females. As for problem drinkers and probable ADS, 13 was the best cut-off point for both sexes. In relation to AUDIT-C, 5 and 4 were the best cut-off points for males and females with high-risk patterns, respectively. The criterion validity of AUDIT and AUDIT-C to detect binge drinking episodes was found to have a moderate $\mathrm{K}$ value. The results obtained show that AUDIT has good psychometric properties to detect early alcohol abuse disorders in university students; however, it is recommended that the cut-off point be reduced to 8 in men. AUDIT-C improves its predictive value by raising the cut-off point by one unit. Items 2 and 3 should be reviewed to increase its predictive value for $\mathrm{BD}$. Keywords: Alcohol-Related Disorders/diagnosis*, Psychometrics/ methods*, University students, AUDIT, AUDIT-C.
\end{abstract}


A UDIT (Alcohol Use Disorders Identification Test) es un cuestionario autoadministrado, corto, fácil de utilizar y flexible, consistente con las definiciones de la CIE-10 de consumo y dependencia al alcohol, y evalúa el consumo en el último año, proporcionando información útil para dar retroalimentación a los encuestados. Por ello, la OMS recomienda su uso sistemático como test de cribado, siendo uno de los más utilizados mundialmente, tanto en contextos sanitarios como no sanitarios (Rosón, 2008).

La versión española ha sido validada en contexto sanitario (Martínez 1996; Rubio, Bermejo, Caballero y Santo-Domingo, 1998) e incluso en población universitaria (Adewuya, 2005; Kokotailo et al., 2004) pero en contextos culturales y con terminología diferente, por lo que no se sabe si sus propiedades psicométricas lo hacen un instrumento adecuado en población universitaria española.

El AUDIT consta de 10 preguntas, las tres primeras se refieren al consumo de riesgo, las tres siguientes exploran posibles síntomas de dependencia y las cuatro últimas evalúan el consumo perjudicial. La puntuación final permite identificar, en función del punto de corte, tanto los consumos de riesgo como la posible dependencia alcohólica. En la validación española (Rubio et al., 1998), el punto de corte para varones fue de 9 o más y en mujeres en 6 o más para la detección del consumo de riesgo. Existe una versión abreviada, el AUDIT-C (que agrupa las tres primeras preguntas), cuya sensibilidad y especificidad confirman su validez para identificar consumo de riesgo.

El interés de contar con un instrumento de detección en población universitaria se debe al cambio que ha experimentado el patrón de consumo en este sector; así, diversos estudios constatan diferencias en la manera de beber de los jóvenes en relación a la formación académica, de manera que aunque los no universitarios beben con más frecuencia (Dawson, Grant, Stinson y Chou, 2004; Kypri y McAnally, 2005), los que cursan estudios superiores tienden a consumir el alcohol en exceso cuando lo hacen (O’Malley y Johnston, 2002). El consumo intensivo de alcohol o binge drinking $(B D)$, constituye uno de los principales comportamientos de riesgo de los universitarios (Cortés, Espejo y Giménez, 2008; Kypri et al., 2009; Wicki, Kuntsche y Gmel, 2010), lo que puede implicar la presencia de problemas a corto y largo plazo. Estudios recientes en jóvenes consumidores de alcohol en elevadas cantidades demuestran cambios fisiológicos en los parámetros de la tensión arterial y en la ansiedad estado inmediatos al consumo (Vinader-Caerols, Monleón y Parra, 2014), y alteraciones estructurales de las sustancia blanca y gris, hiper/hipoactivación neuronal y alteraciones cognitivas en la memoria, atención y ejecución asociados al patrón de BD mantenido en el tiempo (López-Caneda et al., 2014). Aunque existe controversia internacional sobre la definición del término BD, en España el último informe de 2011 del Observatorio Español de la Droga y las Toxico- manías lo define como el que se concentra en sesiones de pocas horas, principalmente en noches de fin de semana, en grupos de iguales y con escasa percepción de riesgo. La prevalencia de BD en jóvenes entre 20 y 29 años según el estudio EDADES 2011/12 es del 15,2\%.

Por todo ello, es importante disponer de instrumentos que permitan la detección precoz y orienten las intervenciones preventivas, de ahí el interés por conocer en detalle la sensibilidad, especificidad y valor predictivo positivo del AUDIT y AUDIT-C en población universitaria, e igualmente determinar la utilidad y validez de estos test.

Las hipótesis que se plantean en este estudio son: En primer lugar, que el cuestionario AUDIT aplicado a población universitaria es un buen instrumento para detectar precozmente problemas de consumo de alcohol y discriminar consumos de riesgo bajo, moderado y elevado; en segundo lugar, que la versión breve (AUDIT-C) tiene una adecuada fiabilidad y validez diagnóstica en población universitaria con consumos de riesgo.

El objetivo de este estudio es determinar las propiedades psicométricas del AUDIT y AUDIT-C para la detección de problemas relacionados con el consumo de alcohol en población universitaria.

\section{Método}

\section{Participantes}

Cumplimentaron los cuestionarios un total de 1352 estudiantes seleccionados mediante muestreo proporcional, y se eliminaron 43 de ellos por no cumplir criterios mínimos de calidad, quedando una muestra de 1309 (un 59,1\% mujeres), con una edad media de 21,78 años $(D S \pm 4,45)$, con un rango de edad de 18 a 65 años. Se consideró como frecuencia esperada de consumo en el último mes el $63 \%$ y un error absoluto del 2,5\% (Consejería para la Igualdad y Bienestar Social de la Junta de Andalucía, 2009).

\section{Instrumentos}

Todos los participantes cumplimentaron un autoinforme anónimo, con variables sociodemográficas, el cuestionario AUDIT y el Diario de consumo semanal de alcohol.

El AUDIT es un cuestionario autoadministrado cuyo rango de puntuación oscila entre 0 y 40 puntos. Las ocho primeras preguntas se puntúan de 0 a 4 y las preguntas nueve y diez con 0,2 y 4 puntos. Los puntos de corte propuestos por Rosón (2008) identifican tres subtipos de poblaciones: bebedor sin problemas (puntos de corte de 0-7 en hombres y 0-5 en mujeres), bebedor de riesgo (puntos de corte de 8-12 y 6-12) y bebedor con problemas físico-psíquicos y probable dependencia alcohólica (SDA) (punto de corte de 13 para ambos). Las tres primeras preguntas de este test conforman el AUDIT-C, que considera consumo de riesgo los valores de cuatro o más en hombres (Bush, Kivlahan, McDonell, Fihn y Bradley, 1998) y tres o más en mujeres (Bradley et al., 2003). 
El Diario de consumo semanal empleado registra el número de Unidades de Bebida Estándar (UBE) consumidas en varios momentos del día u ocasión (mañana, aperitivo, comida, tarde, cena, noche). Según Rodríguez-Martos (2007a), se consideran tres categorías: Consumo de riesgo bajo (número de $\mathrm{UBE} \leq 21 \mathrm{y} \leq 14$ en hombres y mujeres respectivamente), Consumo de riesgo moderado (22-27 UBE y 15-16 UBE) y Consumo de riesgo elevado ( $\geq 28 \mathrm{UBE}$ y $17 \mathrm{UBE}$ ). Se prestó especial atención a la presencia de $B D$, considerado como la ingesta de cinco o más UBE en una sola ocasión, como categoría independiente de riesgo que pudiera reflejar formas de beber problemáticas (Rodríguez-Martos, 2007b).

\section{Procedimientos}

Para estudiar las evidencias de validez y estructura interna de las puntuaciones del AUDIT y AUDIT-C en esta población, se utilizó como patrón de referencia el Diario de consumo semanal. Este diario no solo registra la cantidad, frecuencia, tipología de las bebidas y los patrones de consumo, sino que su interpretación permite identificar y tipificar a las poblaciones de riesgo, así como el $\mathrm{BD}$.

\section{Análisis de datos}

Para el análisis de fiabilidad y validez del AUDIT se utilizaron la versión 20 del paquete estadístico SPSS y el programa FACTOR 9.2.
Fiabilidad. Como medida de la consistencia interna se empleó el coeficiente alfa de Cronbach. Para determinar la correlación entre las diferentes subescalas y la puntuación global se utilizó el coeficiente de correlación de Spearman.

La validez de contenido se evaluó mediante la comparación de las puntuaciones medias en dos grupos de estudiantes con consumos de alcohol significativamente diferentes (consumos de riesgo moderado y elevado). Igualmente se compararon las puntuaciones de cada ítem del AUDIT con las puntuaciones medias obtenidas en el Diario de consumo en cada uno de los tres grupos de estudiantes con consumos de alcohol de riesgo bajo, medio y elevado.

La validez de estructura interna se analizó mediante Análisis Factorial Exploratorio (AFE) para evaluar la dimensionalidad subyacente con valor psicológico, teniendo en cuenta que la versión original del AUDIT ya contempla 3 dimensiones o factores. Mediante el programa FACTOR 9.2 (Lorenzo-Seva y Ferrando, 2006) se analizó si en esta muestra representativa de la población universitaria, la estructura obtenida es coincidente con la estructura teórica. Como método para determinar el número de dimensiones se utilizó el Minimum Average Partial (MAP) (Velicer, 1976); como método de extracción el Unweighted Least Squares (ULS) con rotación Promin; y como método de rotación más eficiente para obtener los puntos de corte idóneos, la rotación Simplimax.

Tabla 1. Patrones de consumo en universitarios (población total y bebedores), por sexo.

\begin{tabular}{|c|c|c|c|c|c|}
\hline \multicolumn{3}{|c|}{ Consumo semanal de alcohol y patrones de consumo } & \multirow{2}{*}{$\begin{array}{l}\text { Hombres } \\
73,1(535)\end{array}$} & \multirow{2}{*}{$\begin{array}{l}\text { Mujeres } \\
64,6(774)\end{array}$} & \multirow{2}{*}{$\begin{array}{l}\text { Total } \\
68,1(1309)\end{array}$} \\
\hline $\begin{array}{l}\text { Población total } \\
(\mathrm{N}=1309)\end{array}$ & $\begin{array}{l}\text { Consumo } \\
\text { última semana }\end{array}$ & $\%(n)$ & & & \\
\hline & \multirow{2}{*}{$\begin{array}{l}\text { UBE } \\
\text { última semana } \\
\text { Media (DS) }\end{array}$} & Jueves & $1,25(3,0)$ & $0,79(2,1)$ & $0,98(2,5)$ \\
\hline & & Viernes a Domingo & $8,14(9,3)$ & $5,30(6,3)^{\star \star}$ & $6,29(8,0)$ \\
\hline & \multirow{3}{*}{$\begin{array}{l}\text { Patrón } \\
\text { Consumo Semanal } \\
(\%)\end{array}$} & Consumo riesgo bajo ${ }^{a}$ & 77,2 & 77,4 & 77,3 \\
\hline & & Consumo riesgo moderado ${ }^{\mathrm{b}}$ & 15,0 & 16,9 & 16,1 \\
\hline & & Consumo riesgo elevado ${ }^{c}$ & 7,9 & 5,7 & 6,6 \\
\hline & Consumo intensivo alcohol (BD) & $\geq 5$ UBE / una ocasión & 38,3 & $32,7^{\star \star \star}$ & 35,0 \\
\hline \multirow[t]{9}{*}{$\begin{array}{l}\text { Población bebedora } \\
(n=891)\end{array}$} & $\begin{array}{l}\text { Consumo } \\
\text { última semana }\end{array}$ & $\%(n)$ & $73,1(391)$ & $64,6(500)$ & $68,1(891)$ \\
\hline & \multirow{4}{*}{$\begin{array}{l}\text { UBE } \\
\text { última semana } \\
\text { Media (DS) }\end{array}$} & Lunes a Miércoles & $2,18(4,1)$ & $0,80(2,3)^{\star \star \star}$ & $1,41(3,3)$ \\
\hline & & Jueves & $1,71(3,4)$ & $1,22(2,6)$ & $1,44(2,9)$ \\
\hline & & Viernes a Domingo & $11,13(9,2)$ & $8,20(6,2)^{\star}$ & $9,49(7,8)$ \\
\hline & & Total semanal & $15,03(12,5)$ & $10,22(8,0)^{\star \star \star}$ & $12,33(10,5)$ \\
\hline & \multirow{3}{*}{$\begin{array}{l}\text { Patrón } \\
\text { Consumo Semanal } \\
\text { (\%) }\end{array}$} & Consumo riesgo bajo ${ }^{a}$ & 68,8 & 65,0 & 66,7 \\
\hline & & Consumo riesgo moderado ${ }^{\mathrm{b}}$ & 20,5 & 26,2 & 23,7 \\
\hline & & Consumo riesgo elevadoc & 10,7 & 8,8 & 9,7 \\
\hline & Consumo intensivo alcohol (BD) & $\geq 5$ UBE / una ocasión & 52,4 & $50,6^{\star \star \star}$ & 51,4 \\
\hline
\end{tabular}

Nota. UBE (Unidad Bebida Estándar); DS (Desviación Estándar); BD (Binge Drinking o consumo intensivo alcohol). ${ }^{a} \leq 21$ UBE hombre y $\leq 14$ UBE mujer. ${ }^{b} 22-27$ UBE hombre y 15-16 UBE mujer. ${ }^{c} \geq 28$ UBE hombre $y \geq 17$ UBE mujer. ${ }^{\star} p=, 018 ;{ }^{\star \star} p=, 002 ;{ }^{\star \star \star} p=, 000$. 
Realizamos un contraste multivariado de la varianza (MANOVA) de las respuestas al AUDIT en las tres categorías de consumos de riesgo determinados a través del diario de consumo semanal de alcohol (en UBE).

La validez de criterio o la validez diagnóstica de las puntuaciones de los cuestionarios AUDIT y AUDIT-C se evaluó a partir de los valores obtenidos con el Diario de consumo utilizado como estándar de referencia. La sensibilidad y especificidad de cada uno de los ítems fueron calculadas para las tres categorías de consumo de riesgo (bajo, medio y elevado) y para el BD. Se obtuvieron las curvas ROC y consideraron varios puntos de corte para identificar la sensibilidad y es-

Tabla 2. Problemas consumo de alcohol según AUDIT (población total y bebedores), por sexo.

\begin{tabular}{|c|c|c|c|c|}
\hline \multicolumn{2}{|l|}{ AUDIT } & $\begin{array}{l}\text { Hombres } \\
\mathrm{N}(\%)\end{array}$ & $\begin{array}{l}\text { Mujeres } \\
\mathrm{N}(\%)\end{array}$ & $\begin{array}{l}\text { Total } \\
\text { N (\%) }\end{array}$ \\
\hline \multirow{4}{*}{$\begin{array}{l}\text { Población } \\
\text { total } \\
(\mathrm{N}=1309)\end{array}$} & $\begin{array}{l}\text { Bebedor sin } \\
\text { problemas }^{a}\end{array}$ & $390(72,9)$ & $572(73,9)$ & $962(73,5)$ \\
\hline & $\begin{array}{l}\text { Bebedor } \\
\text { de riesgo }^{b}\end{array}$ & $101(18,9)$ & $162(20,9)$ & $263(20,1)$ \\
\hline & $\begin{array}{l}\text { Bebedor } \\
\text { con probable } \\
\text { SDA }^{c}\end{array}$ & $44(8,2)$ & $40(5,2)$ & $84(6,4)$ \\
\hline & Total & $535(100,0)$ & $774(100,0)$ & $1309(100,0)$ \\
\hline \multirow{4}{*}{$\begin{array}{l}\text { Población } \\
\text { bebedora } \\
(\mathrm{n}=891)\end{array}$} & $\begin{array}{l}\text { Bebedor sin } \\
\text { problemas }^{a}\end{array}$ & $249(63,7)$ & $304(60,8)$ & $553(62,1)$ \\
\hline & $\begin{array}{l}\text { Bebedor } \\
\text { de riesgo }\end{array}$ & $98(25,1)$ & $156(31,2)$ & $254(28,5)$ \\
\hline & $\begin{array}{l}\text { Bebedor } \\
\text { con probable } \\
\text { SDA }^{c}\end{array}$ & $44(11,3)$ & $40(8,0)$ & $84(9,4)$ \\
\hline & Total & $391(100,0)$ & $500(100,0)$ & $891(100,0)$ \\
\hline
\end{tabular}

Nota. SDA (Síndrome Dependencia Alcohólica).

${ }^{\mathrm{a}} \leq 7$ hombre $\mathrm{y} \leq 5$ mujer. ${ }^{\mathrm{b}}$ 8-12 hombre y 6-12 mujer. ${ }^{\mathrm{c}} \geq 13$ hombre y mujer. pecificidad óptimas. La eficiencia de estos puntos de corte se evaluó mediante el Índice de Youden, y la concordancia entre observaciones mediante el índice Kappa utilizando los criterios de Altman para su clasificación. Para evaluar la validez de criterio de las puntuaciones de AUDIT y AUDIT-C para el diagnóstico de BD se consideró como patrón de referencia el registro de consumos de cinco o más UBE en una sola ocasión.

\section{Resultados}

\section{Patrones de consumo de alcohol}

Según el Diario consumo semanal el 68,1\% de los estudiantes consumió alcohol en la última semana, siendo más frecuente en hombres $(73,1 \%)$ que en mujeres $(64,6 \%)$. Centrando la atención exclusivamente en los estudiantes con consumos en la última semana $(\mathrm{n}=891)$ la prevalencia de Consumo de riesgo moderado fue del $23,7 \%$ y de $9,7 \%$ el elevado. La ingesta media semanal fue de 15,03 UBEs en los hombres, con valores significativamente inferiores $(\mathrm{p}=, 000)$ en las mujeres $(10,22 \mathrm{UBE} /$ semana) (Tabla 1$)$.

El BD estuvo presente independientemente de que el patrón de consumo semanal fuese de riesgo bajo, moderado o elevado; así, un 3,9\% de hombres y un $4.0 \%$ de mujeres bebieron de esta manera a pesar de que el consumo semanal fue considerado de riesgo bajo.

Según el AUDIT, una cuarta parte $(26,5 \%)$ de la muestra presentó problemas de consumo de alcohol, de los cuales el $20,1 \%$ son Bebedores de riesgo y el 6,4\% Bebedores con problemas físico-psíquicos y probable SDA. En el grupo de consumidores en la última semana estos valores aumentan hasta el 28,5\% y el 9,4\% respectivamente (Tabla 2 ).

En cuanto al AUDIT-C, más de la mitad de los bebedores $(63,2 \%)$ alcanza los puntos de corte para el Consumo de riesgo con predominio $(\mathrm{p}=, 000)$ en el sexo femenino $(26,8 \%$ hombres vs $36,4 \%$ mujeres).

Tabla 3. Correlación de las puntuaciones de cada ítem del AUDIT con el total de la escala.

\begin{tabular}{llllll}
\hline AUDIT & Media & DS & $\begin{array}{l}\text { Matriz de correlación } \\
\text { inter-elementos }\end{array}$ & $\begin{array}{l}\text { Matriz de covarianza } \\
\text { inter-elementos }\end{array}$ & $\begin{array}{l}\text { Alfa de Cronbach si } \\
\text { elimina el elemento }\end{array}$ \\
\hline Ítem 1 & 1,68 & 1,001 & 0,706 & 2,960 & 0,709 \\
Ítem 2 & 0,64 & 0,856 & 0,662 & 2,372 & 0,718 \\
Ítem 3 & 0,66 & 0,938 & 0,811 & 3,187 & 0,700 \\
Ítem 4 & 0,29 & 0,719 & 0,610 & 1,836 & 0,726 \\
Ítem 5 & 0,18 & 0,448 & 0,602 & 1,130 & 0,736 \\
Ítem 6 & 0,12 & 0,410 & 0,503 & 0,864 & 0,741 \\
Ítem 7 & 0,30 & 0,592 & 0,593 & 1,471 & 0,731 \\
Ítem 8 & 0,37 & 0,638 & 0,694 & 1,855 & 0,723 \\
Ítem 9 & 0,10 & 0,549 & 0,414 & 0,951 & 0,742 \\
Ítem 10 & 0,11 & 0,593 & 0,369 & 0,918 & 0,744 \\
\hline TOTAL & 4,45 & 4,188 & 1,000 & 17,542 & 0,800 \\
\hline
\end{tabular}




\section{Estudio de la fiabilidad}

Consistencia interna. La medida de consistencia interna (alfa de Cronbach) del AUDIT aporta un resultado de 0,75; por dominios, la subescala Consumo de riesgo obtuvo un valor de 0,83 , Sintomas de Dependencia un 0,79, y Consumo perjudicial un valor de 0,75 .

Las puntuaciones más elevadas fueron las de las preguntas $1^{\mathrm{a}}, 2^{\mathrm{a}}$ y $3^{\mathrm{a}}$ que constituyen el dominio Consumo de riesgo, y que exploran frecuencia y cantidad de consumo de alcohol. En cambio, los ítems $5^{\circ}, 6^{\circ}, 9^{\circ}$ y $10^{\circ}$ obtuvieron los valores menores, siendo estos los que recogen información sobre presencia de sintomatología de dependencia, lesiones sufridas por el alcohol y preocupación de otros por el consumo que la persona lleva a cabo (Tabla 3 ).

Fiabilidad AUDIT. Diario de consumo semanal de alcohol. Utilizando el Diario de consumo semanal de alcohol como patrón de referencia frente al AUDIT, se obtuvieron resultados concordantes en más del $90 \%$ de los casos. Respecto a la fiabilidad, el coeficiente de correlación de Spearman fue del 0,87 , lo que indica una buena concordancia. El valor de Kappa fue de 0,85, considerándose según los criterios de Altman como muy bueno (Tabla 4).

Fiabilidad AUDIT-C. Diario de consumo semanal de alcohol. Utilizando la versión breve, AUDIT-C, frente al diario de consumo semanal de alcohol medido en UBEs, la fiabilidad fue también buena o moderada. El coeficiente de correlación de Spearman fue 0,57 , indicativo de una concordan- cia buena. El valor de Kappa es de 0,50, moderado, según la valoración de Altman (Tabla 5).

\section{Validez de contenido}

Se seleccionaron para este análisis a los estudiantes con Consumo de riesgo moderado y elevado. La puntuación media del AUDIT en el grupo con Consumo de riesgo moderado fue de 9,45 para los hombres y de 7,92 para las mujeres, mientras que la puntuación media del AUDIT para aquellos con Consumo de riesgo elevado fue de 15,19 para hombres y 13,09 para mujeres. Se detectan puntuaciones medias diferentes en el AUDIT con valores estadísticamente significativas para los distintos tipos de consumo $(\mathrm{p}<, 001)$; por tanto, el contenido del mismo se muestra válido para discriminar grupos de individuos con diferentes consumos.

$\mathrm{Al}$ analizar las puntuaciones por ítems en los distintos grupos de riesgo, éstas aumentan gradualmente desde el grupo de riesgo bajo al elevado, pasando por el moderado, en función de los distintos tipos de consumo, salvo en el ítem 2. El ítem 2 dice: ¿¿Cuántas consumiciones de bebidas alcohólicas suele realizar en un día de consumo normal?, siendo las posibles puntuaciones: 0 (una o 2), 1 (tres o 4), 2 (Cinco o 6), 3 (de siete a 9) o 4 (diez o más). En esta pregunta la puntuación media del grupo con riesgo moderado es mayor que la del grupo de riesgo alto $(1,81$ vs 1,64$)$. La opción consumir "cinco o seis bebidas" en el grupo con Consumo de riesgo moderado representa una media de 24,18 UBE/semana y en el grupo con consumo de riesgo elevado de 38,74 UBE/semana, lo que significaría que a igualdad de número de consumiciones (5 ó 6), es mayor la ingesta de alcohol (número de UBE) en un grupo que en el otro.

Tabla 4. Fiabilidad del AUDIT frente al Diario de Consumo Semanal de Alcohol.

\begin{tabular}{|c|c|c|c|c|c|c|}
\hline & & & \multicolumn{4}{|c|}{ Diario de consumo } \\
\hline & & & $\begin{array}{l}\text { Consumo } \\
\text { riesgo bajo }\end{array}$ & $\begin{array}{c}\text { Consumo } \\
\text { riesgo moderado }\end{array}$ & $\begin{array}{c}\text { Consumo } \\
\text { riesgo elevado }\end{array}$ & Total \\
\hline \multirow[t]{4}{*}{ AUDIT } & Bebedor sin problemas $^{d}$ & n (\%) & $952(54,1)$ & $6(2,8)$ & $4(4,7)$ & $962(73,5)$ \\
\hline & Bebedor de riesgo ${ }^{e}$ & n (\%) & $55(5,4)$ & $204(96,7)$ & $4(4,7)$ & $263(20,1)$ \\
\hline & Bebedor con probable SDA ${ }^{f}$ & n (\%) & $5(0,5)$ & $1(0,5)$ & $78(90,7)$ & $84(6,4)$ \\
\hline & Total & n (\%) & $1012(100)$ & $211(100)$ & $86(100)$ & $1309(100)$ \\
\hline
\end{tabular}

a $\leq 21$ UBE hombre $y \leq 14$ UBE mujer. ${ }^{b} 22-27$ UBE hombre y $15-16$ UBE mujer. ${ }^{c} \geq 28$ UBE hombre y $\geq 17$ UBE mujer. ${ }^{d} \leq 7$ hombre y $\leq 5$ mujer. ${ }^{\text {e }} 8-12$ hombre y $6-12$ mujer. ${ }^{\mathrm{f}} \geq 13$ hombre y mujer. *Correlación de Spearman $(0,87)$, Kappa $(0,85)$.

Tabla 5. Fiabilidad del AUDIT-C frente al Diario de Consumo Semanal de Alcohol.

\begin{tabular}{|c|c|c|c|c|c|}
\hline & & & \multicolumn{3}{|c|}{ Diario de consumo } \\
\hline & & & $\begin{array}{l}\text { Consumo } \\
\text { riesgo bajo }^{\text {a }}\end{array}$ & $\begin{array}{l}\text { Consumo riesgo } \\
\text { moderado y elevado }\end{array}$ & Total \\
\hline \multirow[t]{3}{*}{ AUDIT-C } & Consumo sin problemas ${ }^{c}$ & n (\%) & $705(69,7)$ & $4(1,3)$ & $709(54,2)$ \\
\hline & Consumo de riesgo ${ }^{d}$ & n (\%) & $307(30,3)$ & $293(98,7)$ & $600(45,8)$ \\
\hline & Total & n (\%) & $1012(100)$ & $297(100)$ & $1309(100)$ \\
\hline
\end{tabular}

${ }^{\mathrm{a}} \leq 21$ hombre $\mathrm{y} \leq 14$ mujer. ${ }^{\mathrm{b}} \geq 22$ hombre $\mathrm{y} \geq 15$ mujer. ${ }^{\mathrm{c}} \leq 3$ hombre $\mathrm{y} \leq 2$ mujer. ${ }^{\mathrm{d}} \geq 4$ hombre $\mathrm{y} \geq 3$ mujer. ${ }^{*}$ Correlación de Spearman (o,57), Kappa (o,50) 
Tabla 6. Análisis factorial exploratorio del AUDIT.

\begin{tabular}{l|cc|ccc}
\hline AUDIT & \multicolumn{2}{|c|}{ Dos factores } & \multicolumn{3}{|c}{ Tres factores } \\
& Factor 1 & Factor 2 & Factor 1 & Factor 2 & Factor 3 \\
\hline Ítem 1 & & 0,957 & & 0,647 & \\
Ítem 2 & & 0,742 & & 0,615 & \\
Ítem 3 & & 1,028 & & 1,081 & \\
Ítem 4 & & 0,560 & 0,618 & & \\
Ítem 5 & & 0,718 & 1,012 & & \\
Ítem 6 & & 0,591 & 0,717 & & \\
Ítem 7 & & 0,554 & 0,498 & & \\
Ítem 8 & & 0,761 & & 0,584 & \\
Ítem 9 & 0,832 & & & & 0,937 \\
Ítem 10 & 0,957 & & & & 0,693 \\
\hline Fiabilidad & & & & & \\
estimada & 0,825 & 0,923 & 0,870 & 0,925 & 0,833 \\
\hline
\end{tabular}

\section{Validez de estructura interna}

$\mathrm{Al}$ analizar si en nuestra población se reproducen las tres dimensiones originales del AUDIT se obtienen tres factores; el primero formado por los ítems 4, 5, 6 y 7 con una fiabilidad del 0,87; el segundo factor integrado por los ítems 1, 2 , 3 y 8 con una fiabilidad del 0,92 ; y finalmente un tercer factor que agrupa los ítems 9 y 10 con una fiabilidad de 0,83 . La magnitud media de las correlaciones residuales (RMCR) fue de 0,02 valor que considera que el ajuste del modelo es aceptable, aunque la asociación de ítems encontrada difiere de la original.

$\mathrm{Al}$ considerar dos dimensiones encontramos una primera formada por los 8 primeros ítems, Consumo de riesgo, y una segunda que agrupa los 2 últimos, Consumo perjudicial, que explicarían el $66 \%$ de la varianza acumulada y con una fiabilidad estimada del 0,82 y 0,92 respectivamente. La media de correlaciones residuales fue de 0.03 (Tabla 6 ).

Los diferentes niveles de riesgo de los consumos de alcohol tienen su reflejo en las respuestas a las preguntas del AUDIT (3, 5 y 8, especialmente según MANOVA). La variabilidad no explicada por las diferencias de grupo fue del $32 \%$ en varones (Wilks $\mathrm{F} 42,8 \mathrm{p}<0,001$ ) y $25 \%$ en mujeres (F75,6 p <0,001). Todas las preguntas del AUDIT resultaron significativas para discriminar entre diferentes consumos de riesgo.

\section{Validez de criterio}

Se han comparado los diagnósticos proporcionados por AUDIT y AUDIT-C con los valores de ingesta de alcohol semanal utilizado como patrón de referencia. La validez de criterio del AUDIT para Bebedor sin problemas, Bebedor de riesgo, y Bebedor con problemas físico-psíquicos y probable SDA presenta un valor $K$ alto para ambos sexos $(0,83 ; 0,83 ; 0,92$ en hombres y 0,$88 ; 0,88 ; 0,90$ en mujeres); la validez de criterio para las tres categorías de bebedores muestra una fuerza de concordancia muy buena, con resultados aún mejores para el sexo femenino en el subgrupo de Bebedoras de riesgo.

Los valores de sensibilidad en el AUDIT-C fueron del $98 \%$ para hombres y mujeres, ahora bien, los valores $K$ obtenidos para ambos sexos fueron moderados $(0,49$ y 0,51 respectivamente) según los criterios de Altman.

\section{Curvas ROC}

Con respecto al AUDIT, el mejor equilibrio entre sensibilidad y especificidad para la detección de Bebedor de riesgo se obtuvo para un punto de corte de 7,50 para los hombres (sensibilidad de $95 \%$ y especificidad de $93 \%$ ), y de 5,50 para las mujeres (sensibilidad de $98 \%$ y especificidad de $95 \%$ ). Respecto al Bebedor con problemas físico-psíquicos y probable SDA este fue de 12,50 para ambos sexos (sensibilidad de 95\% para los hombres y $86 \%$ para las mujeres y una especificidad de $99 \%$ para ambos).

El área bajo la curva ROC mostró, en el caso de Bebedor de riesgo un rendimiento muy bueno en hombres $(0,963)$ y excelente para las mujeres $(0,976)$. Para Bebedor con problemas y probable SDA el área bajo la curva resultó excelente en ambos sexos (0,983 hombres y 0,973 mujeres). En cuanto a la validez, los resultados mostraron valores del área bajo la curva ROC cercanos a uno en ambas categorías cuando comparamos la puntuación con el Diario de consumo.

Para el AUDIT-C el mejor equilibrio entre sensibilidad y especificidad para Consumo de riesgo se encontró para la puntuación de 4,50 para hombres (sensibilidad de $91 \%$ y especificidad de $84 \%$ ), y de 3,50 para mujeres (sensibilidad de $86 \%$ y especificidad de $88 \%$ ). El área bajo la curva atendiendo al sexo lo identificó como un test muy bueno $(0,941$ hombres y 0,945 mujeres). Respecto a su validez, los resultados mostraron valores cercanos a uno para la categoría estudiada cuando se comparó su puntuación con el Diario de consumo.

\section{Análisis de los puntos de corte}

Los puntos de corte óptimos para AUDIT para las categorías Bebedor de riesgo y Bebedor con problemas físico-psíquicos y probable SDA fueron de 8 y 13 para varones, y de 6 y 13 para mujeres, respectivamente. El mejor punto de corte del AUDIT-C para detectar Consumo de riesgo en hombres fue de cinco puntos y para mujeres cuatro (Tabla 7).

\section{Discusión}

Las características sociodemográficas de la muestra son similares a las de otros estudios sobre consumo de alcohol en universitarios (Adewuya, 2005; Kokotailo et al., 2004; Londoño, García, Valencia y Vinaccia, 2005; Martín-Montañez et al., 2011; Montaño, Morales, Gómez, Vera y Gantiva, 2011; Salazar, Ávila, Pérez y Martínez, 2010; Seguel, Santander y Alexandre, 2013; Zaldívar, López, García y Molina, 2011), con un discreto predominio de mujeres participan- 
tes $(59.01 \%)$ y una edad media de 21,78 años. Un tercio no vive en el domicilio familiar, siendo este grupo el que presenta consumos de riesgo moderado-elevados más altos, porcentajes mayores de bebedores de riesgo, y con problemas físico-psíquicos y probable SDA. Este hecho pone de manifiesto la conveniencia de considerar el tipo de convivencia que tienen los universitarios a la hora de diseñar medidas preventivas para ellos.

La estimación de la fiabilidad de las puntuaciones del AUDIT mediante el alfa de Cronbach fue buena, tanto globalmente como para cada una de las subescalas, lo que confirma su idoneidad para el cribado de problemas por consumo de alcohol en el contexto universitario. Los valores de fiabilidad obtenidos son muy parecidos a los de una revisión sistemática que incluyó 10 estudios en distintos contextos (Meneses-Gaya, Waldo, Regina y Crippa, 2009), a los referidos en muestras de población general (Alvarado, Garmendia, Acuña, Santis y Arteaga, 2009; Contel, Gual y Colom, 1999; Rubio et al., 1998), y en dos muestras de universitarios americanos (Kokotailo et al., 2004) y chilenos (Seguel et al., 2013), donde se obtienen valores medios de 0,80 .

El hecho de que se obtuvieran las puntuaciones más altas en los ítems 1, 2 y 3 (exploran cantidad y frecuencia del alcohol consumido) y las más bajas en los 5, 6, 9 y 10 (miden las consecuencias del consumo, ya sea dependencia, lesiones o recriminación del entorno), es indicativo de abuso de alcohol, lo que concuerda con el cambio de patrón del consumo en los jóvenes con un protagonismo destacado de la práctica del atracón (BD) por una parte, y por otra con las menores prevalencias de dependencia alcohólica previsibles en este sector de población.
Según los resultados de la subescala Consumo perjudicial del AUDIT la prevalencia de estudiantes que expresó dificultades para abandonar el consumo fue $6,4 \%$ para la muestra total y $9,4 \%$, para el grupo de bebedores; esto pondría de manifiesto que hasta una décima parte de la población bebedora universitaria es susceptible de tener SDA. Un estudio en universitarios chilenos (Baader et al., 2014) habla sólo de un $1,5 \%$ de probable SDA.

Respecto al ítem 2 del AUDIT en la opción consumir "cinco o seis bebidas" el consumo semanal medio es mayor en la categoría Consumo de riesgo moderado que en elevado (24,18 UBE/semana vs 38,74 UBE/semana). Este ítem hace referencia a cantidad y no a frecuencia de consumo; al analizar la validez de contenido hay que tener en cuenta que un individuo puede consumir muchas bebidas alcohólicas diariamente pero de baja graduación y otro en cambio el mismo o incluso menor número de bebidas pero de alta graduación. Por otro lado, en la validación del AUDIT al catalán y castellano (Contel et al., 1999) este ítem obtenía diferente resultado a la validación española de Rubio et al. (1998), lo que justificaban como debidas a la traducción. Considerando estas cuestiones parece recomendable revisar el ítem 2, de forma que se analicen separadamente los parámetros frecuencia y/o cantidad de consumo. Además, a la vista de la evolución del concepto de $\mathrm{BD}$, el parámetro frecuencia debería registrarse de forma más específica considerando cuantas bebidas se consumen en una sola ocasión y no en un día completo; y en cuanto a cuantificación, sería interesante incorporar el concepto de UBE.

Respecto al ítem 3 del AUDIT: ¿Con qué frecuencia toma seis o más bebidas alcohólicas en una sola ocasión de consumo?

Tabla 7. Índices de validez y utilidad para los puntos de corte del AUDIT y AUDIT-C.

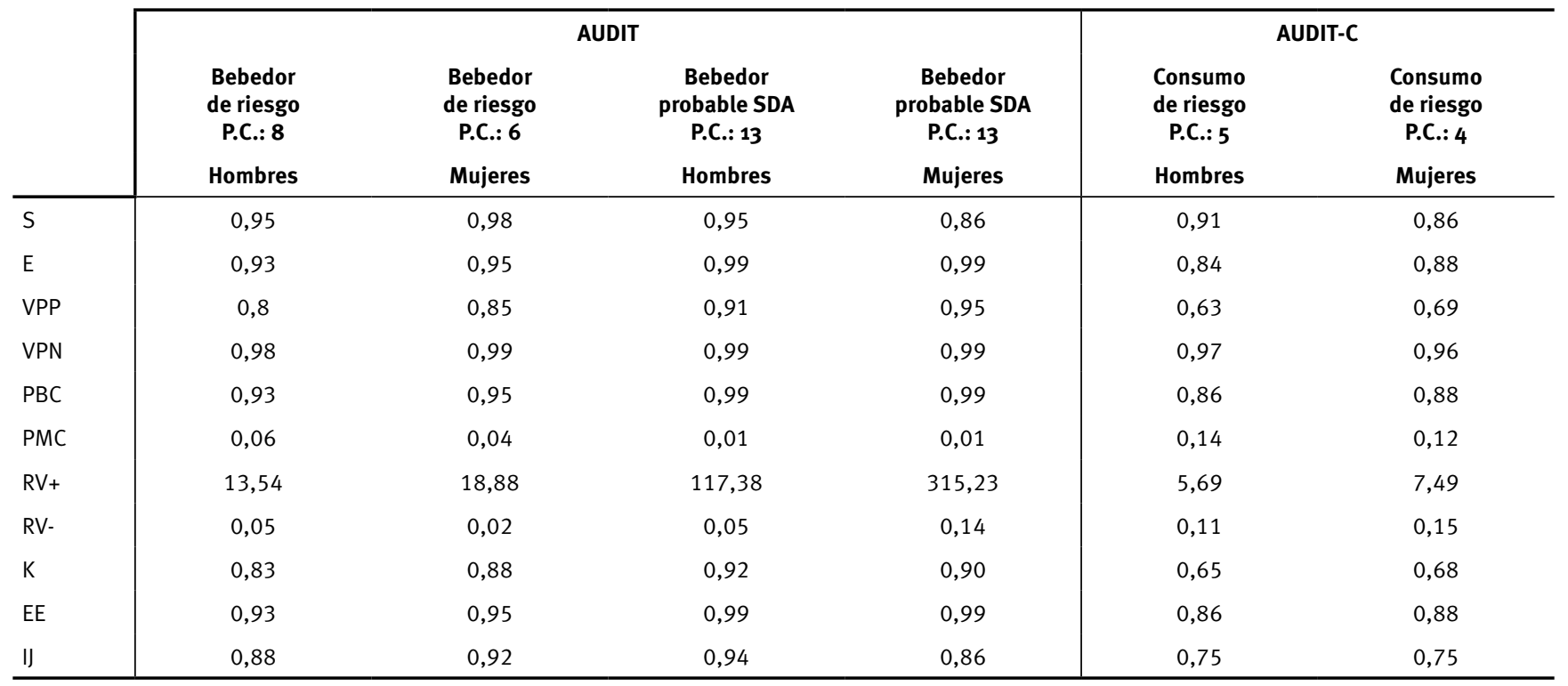

Nota. SDA (Síndrome Dependencia alcohólica); P.C. (Punto de corte); S (Sensibilidad); E (Especificidad); VPP (Valor Predictivo Positivo); VPN (Valor Predictivo Negativo); PBC (Porcentaje de Bien Clasificados); PMC (Porcentaje de Mal Clasificados); RV+ (Razón de Verosimilitud Positiva); RV- (Razón de Verosimilitud Negativa); K (Coeficiente de Kappa); EE (Eficiencia Estándar); IJ (Índice de Youden). 
En la validación en universitarios estadunidenses (Kokotailo et al., 2004) recomienda reducir a cinco o más bebidas para hombres, y a cuatro o más para mujeres. Si recordamos que el BD consiste en ingerir una cantidad de alcohol igual o superior a cinco UBE en una sola ocasión, cabe destacar que estos pueden presentarse independientemente del patrón de consumo. En nuestro estudio al analizar la validez de criterio de aquellos que tienen consumo intensivo con el AUDIT, se obtiene un valor $K$ moderado para ambos sexos (0,54 en hombres vs 0,59 en mujeres), siendo el valor predictivo positivo del AUDIT de $82,8 \%$ en varones y $80,2 \%$ en las mujeres. Teniendo en cuenta lo anterior, sería recomendable revisar la redacción de este ítem y considerar la reducción a cinco bebidas o más en una sola ocasión, o mejor aún incorporar el concepto de cinco UBE, reflejando así con mayor exactitud el concepto de BD.

La estructura original del AUDIT presenta tres dimensiones: consumo de riesgo (ítems 1-3), síntomas de dependencia (ítems 4-6) y consumo perjudicial de alcohol (ítems 7-10). El análisis factorial del AUDIT produce resultados diferentes dependiendo de la prevalencia del problema en la población de estudio.

El modelo factorial de tres dimensiones obtenido, no coincide exactamente con la estructura original ya que los ítems 7 y 8 no se conservan en el dominio de Consumo perjudicial, sino que el ítem 7 se adscribe a Síntomas de probable dependencia y el ítem 8 a Consumo de riesgo.

Consideramos que tiene mayor valor desde el punto de vista del diseño de programas de intervención en población universitaria un modelo de dos factores, en el que con una fiabilidad elevada, las puntuaciones del test AUDIT discriminen entre consumos de riesgos y consumos perjudiciales.

En nuestro estudio, efectivamente los estudiantes universitarios presentaban una prevalencia de problemas de consumo de alcohol del $26,5 \%$ (un $20,1 \%$ pertenece a la categoría de bebedor de riesgo y un 6,4\% a la de bebedor con problemas físico-psíquicos y probable SDA) y se obtuvieron dos factores, que estaban integrados por los ítems 1 al 8 y por los ítems 9 y 10 .

Otros estudios en población general apoyan también una estructura bidimensional (Contel et al., 1999; Lima et al., 2005; Meneses-Gaya et al., 2009). En población universitaria chilena (Seguel et al., 2013) también se determinan dos componentes, que se relacionan con el consumo propiamente y con las consecuencias que acarrea un consumo excesivo.

En cuanto a la validez interna, los resultados de sensibilidad y especificidad obtenidos para el AUDIT (Sensibilidad $=32-96 \%$, y Especificidad $=84-96 \%$ ) se sitúan en el extremo más alto del rango de valores publicados (Barry y Fleming, 1993; Rubio et al., 1998; Saunders, Aasland, Babor, de la Fuente y Grant, 1993; Schmidt, Barry y Fleming, 1995), lo que demuestra su utilidad para el cribado de problemas relacionados con el consumo de alcohol en este sector de población.

Numerosos estudios de validación del AUDIT obtienen una buena sensibilidad y especificidad utilizando diferentes puntos de corte (Adewuya, 2005; Dawson, Grant, y Stinson, 2005; Gache et al., 2005; Knight, Sherritt, Harris, Gates, y Chang, 2003; Kokotailo et al., 2004; Rubio et al., 1998), señalando la necesidad de establecer diferentes puntos de corte en función del sexo, dado que a igualdad de consumo las probables repercusiones del alcohol son más frecuentes en la mujer, por lo que se recomienda reducir el punto de corte a emplear en el sexo femenino de cara a posteriores intervenciones (Reinert y Allen, 2002, 2007).

En las curvas ROC, el área bajo la curva se puede utilizar como indicador de calidad de una prueba diagnóstica, en el caso del AUDIT para la categoría de bebedor de riesgo fue de 0,96 en los hombres y de 0,98 en las mujeres; y para bebedor con problemas físico-psíquicos y probable SDA fue de 0,98 en los hombres y de 0,97 en las mujeres. Estos valores pueden considerarse como muy buenos, y superiores a los obtenidos en la validación española del AUDIT realizada en Atención Primaria (Rubio et al., 1998), en la que se obtiene un área de 0,87 para los problemas por el uso de alcohol y en la validación chilena (Alvarado et al., 2009), con unas áreas de 0,93 para consumo riesgoso, 0,88 en consumo perjudicial y 0,91 para la dependencia. Si lo comparamos con valores de estudios en población universitaria, la validación del AUDIT realizada en la universidad de Wisconsin aportó peores indicadores, con áreas de 0,87 para consumo de alcohol y de 0,77 para abuso o dependencia.

En este estudio los puntos de corte más eficaces para la detección de problemas por el uso de alcohol (bebedor de riesgo) con el AUDIT en hombres fue de ocho (Sensibilidad $=95 \%$, y Especificidad $=93 \%)$ y en mujeres de seis (Sensibilidad $=98 \%$, y Especificidad $=95 \%)$. En la validación española del instrumento (Rubio et al., 1998), se sitúa el punto de corte general para la detección de problemas por el uso de alcohol en ocho, pero cuando analizan la sensibilidad y especificidad por sexos encuentran diferencias, se establecen para hombres nueve o más puntos y en mujeres seis o más. En la validación del AUDIT al catalán y castellano (Contel et al., 1999) también se establece en nueve el punto de corte para hombres, sin llegar a detectar un punto de corte adecuado para el sexo femenino. En otro estudio de validación del AUDIT realizado exclusivamente en mujeres en dos centros de Atención Primaria y un centro de Drogodependencias (Pérula et al., 2005) también se obtuvo un punto de corte para estas de seis o más.

Atendiendo a los resultados obtenidos también sería recomendable reducir el punto de corte para varones en población universitaria en un punto respecto a los valores referenciados en dos estudios de validación en población general realizados en Atención Primaria. Esta recomendación se ve apoyada por una revisión sistemática (Meneses-Gaya 
et al., 2009), que afirma también que la sensibilidad y especificidad del AUDIT son menores cuando se emplean los puntos de corte tradicionales en población universitaria.

Respecto a la detección de bebedores con problemas físico-psiquicos y probable dependencia alcohólica (SDA), el punto de corte más eficaz en este estudio fue de 13 o más para ambos sexos, idéntico al recomendado en atención primaria para la población general (Rosón, 2008).

En cuanto a la validez de criterio del AUDIT-C podría parecer contradictorio que un test que detecta un $98 \%$ de sujetos casos no tenga utilidad para detectar consumos de riesgo, pero hay que tener en cuenta las bajas prevalencias de consumo en población universitaria que hacen que el número de falsos positivos igualen el de verdaderos positivos, por lo que ante un resultado positivo tendríamos las mismas posibilidades de detección, tal y como refleja el cálculo del valor kappa.

A pesar de que la estimación de la fiabilidad del AUDIT-C no fue buena en esta población (índice Kappa 0,50), podría mejorarse para la detección del consumo de riesgo si se aumenta en una unidad sobre el punto de corte recomendado tanto para hombres (Bush et al., 1998) como para mujeres (Bradley et al., 2003). Así, llevando el punto de corte a cinco y cuatro respectivamente, se mejora significativamente su poder predictivo.

La necesidad de detectar el BD en población juvenil viene avalada por el último informe de 2011 del Observatorio Español sobre Drogas, que describe una elevada prevalencia de este patrón de consumo intermitente. Además, según los datos de EDADES 2011/12 asciende ligeramente el consumo de alcohol en forma de atracón en los últimos 30 días; así el $15,2 \%$ de los encuestados ha consumido alcohol en forma de atracón concentrándose especialmente esta forma de consumo de riesgo en el grupo de adultos jóvenes de 20 a 29 años de ambos sexos. La validez de criterio o validez diagnóstica para detectar los BD con el AUDIT es baja, obteniéndose valores de Kappa moderados para ambos sexos. Sin embargo, el AUDIT-C tiene buena sensibilidad para detectar problemas con el alcohol y el consumo intensivo de alcohol tanto en hombres como en mujeres (AUDIT: Sensibilidad de 0,58 en hombres y 0,64 en mujeres vs AUDIT-C: Sensibilidad de 0,81 en hombres y 0,85 en mujeres).

Una posible limitación que puede atribuírsele a este estudio es no haber realizado test-retest, pero fue obviado por razones organizativas y por evitar la percepción que pudieran tener los estudiantes de perder la confidencialidad.

En conclusión, el AUDIT es un instrumento con propiedades psicométricas adecuadas para detectar precozmente problemas de consumo de alcohol en población universitaria, recomendándose disminuir el punto de corte en ocho para varones y revisar los ítems 2 y 3 para aumentar el valor predictivo del consumo intensivo de alcohol. El AUDIT-C mejoraría su validez predictiva al subir en una unidad el punto de corte para ambos sexos.

\section{Reconocimientos}

El estudio se enmarca en un conjunto de actuaciones que lleva la universidad de Cádiz en colaboración con la Junta de Andalucía en materia de prevención de las drogodependencias, por lo que era necesario disponer de un instrumento capaz de discriminar a la población y diseñar actuaciones preventivas acordes a sus resultados.

Se agradece a la Universidad de Cádiz, el apoyo y las facilidades ofrecidas para la realización del trabajo de campo de este estudio; y al Servicio Provincial de Drogodependencias de la Diputación Provincial de Cádiz por su asesoramiento y participación en el protocolo de derivación de los casos detectados.

\section{Conflicto de intereses}

Los autores declaran no tener conflicto de intereses.

\section{Referencias}

Adewuya, A. O. (2005). Validation of the Alcohol Use Disorders Identification Test (AUDIT) as a screening tool for alcohol-related problems among Nigerian university students. Alcohol and Alcoholism, 40, 575-577.

Alvarado, M. E., Garmendia, M. L., Acuña, G., Santis, R. y Arteaga, O. (2009). Validez y confiabilidad de la versión chilena del Alcohol Use Disorders Identification Test (AUDIT). Revista Médica Chile, 137, 1463-1468.

Baader, T., Rojas, C., Molina, J. L., Gotelli, M., Alamo, C., Fierro, C.,... Dittus, P. (2014). Diagnóstico de la prevalencia de trastornos de la salud mental en estudiantes universitarios y los factores de riesgo emocionales asociados. Revista Chilena de Neuro-Psiquiatría, 52, 167-176.

Barry, K. y Fleming, M. (1993). A comparison of the Alcohol Use Disorders Identification Test (AUDIT) to the SMAST-13 in a primary care simple. Alcohol and Alcoholism, 2, 33-42.

Bradley, K. A., Bush, K. R., Epler, A. J., Dobie, D. J., Davis, T. M., Sporleder, J. L.,... Kivlahan, D. R. (2003). Two brief alcohol-screening tests from the Alcohol Use Disorders Identification Test (AUDIT): Validation in a female veterans affairs patient population. Archives of Internal Medicine, 163, 821-829.

Bush, K., Kivlahan, D. R., McDonell, M. S., Fihn, S. D. y Bradley, K. A. (1998). The AUDIT Alcohol Consumption Questions (AUDIT-C): An effective brief screening test for problem drinking. Archives of Internal Medicine, $158,1789-1795$

Consejería para la Igualdad y Bienestar Social de la Junta de Andalucía (2009). La población andaluza ante las drogas XI. Dirección General para las Drogodependencias y Adicciones de Andalucía. Fundación andaluza para la atención a las drogodependencias e incorporación social. 
Contel Guillamon, M., Gual Sole, A. y Colom Farran, J. (1999). Test para la identificación de trastornos por uso de alcohol (AUDIT): traducción y validación del AUDIT al catalán y castellano. Adicciones, 11, 337-347.

Cortés Tomás, M. T., Espejo Tort, B., y Giménez Costa, J. A. (2008). Aspectos cognitivos relacionados con la práctica del botellón. Psicothema, 20, 396-402.

Dawson, D. A., Grant, B. F., Stinson, F. S. y Chou, P. S. (2004). Another look at heavy episodic drinking and alcohol use among college and non college youth. Journal of Studies on Alcohol, 65, 477-489.

Dawson, D. A., Grant, B. F. y Stinson, F. S. (2005). The AUDIT-C: screening for alcohol use disorders and risk drinking in the presence of other psychiatric disorders. Comprehensive Psychiatry, 46, 405-416.

España, Observatorio Español sobre Drogas. Delegación del Gobierno para el Plan Nacional sobre Drogas. Ministerio de Sanidad y Consumo. Informe 2011. Disponible en http://www.pnsd.msc.es/Categoria2/observa/pdf/ oed2011.pdf

Gache, P., Michaud, P., Landry, U., Accietto, C., Arfaoui, S., Wenger, O. y Daeppen, J. B. (2005). The Alcohol Use Disorders Identification Test (AUDIT) as a Screening Tool for Excessive Drinking in Primary Care: Reliability and Validity of a French Version. Alcoholism: Clinical and Experimental Research, 29, 2001-2007.

Knight, J. R., Sherritt, L., Harris, S. K., Gates, E. C. y Chang, G. (2003). Validity of brief alcohol screening test among adolescents: A comparison of the AUDIT, POSIT, CAGE and CRAFFT. Alcoholism: Clinical and Experimental Research, 27, 67-73.

Kokotailo, P. K., Egan, J., Gangnon, R., Brown, D., Marlon, M. y Fleming, M. (2004). Validity of the Alcohol Use Disorders Identification Test in College Students. Alcoholism: Clinical and Experimental Research, 28, 914-920.

Kypri, K. y McAnally, H. M. (2005). Randomized controlled trial of a web-based primary care intervention for multiple health risk behaviors. Preventive Medicine, 41, 761-763.

Kypri, K., Paschall, M. J., Langley, L., Baxter, J., CashellSmith, M. y Bourdeau, B. (2009). Drinking and Alcohol-Related Harm Among New Zealand University Students: Findings From a National Web-Based Survey. Alcoholism: Clinical and Experimental Research 33, 307-314.

Lima, C. T., Freire, A. C., Silva, A. P., Teixeira, R. M., Farrel, M. y Farrel, M. (2005). Concurrent and construct validity of the Audit in urban Brazilian simple. Alcohol and Alcoholism, 40, 584-589.

López-Caneda, E., Mota, N., Greco, A., Velasquez, T., Corral, M., Rodríguez Holguín, S., Cadaveira, F. (2014). Anomalías neurocognitivas asociadas al consumo intensivo de alcohol (binge drinking) en jóvenes y adolescentes: Una revisión. Adicciones, 26, 334-359.

Londoño Pérez, C., García Hernández, W., Valencia Lara, S. C. y Vinaccia Alpi, S. (2005). Expectativas frente al consumo de alcohol en jóvenes universitarios colombianos. Anales de Psicología, 21, 259-287.

López-Caneda, E., Mota, N., Crego, A., Velasquez, T., Corral, M., Rodríguez Holguín, S. y Cadaveira, F. (2014). Anomalías neurocognitivas asociadas al consumo intensivo de alcohol (binge drinking) en jóvenes y adolescentes: Una revisión. Adicciones, 26, 334-359.

Lorenzo-Seva, U. y Ferrando, P. J. (2006). FACTOR: A computer program to fit the exploratory factor analysis model. Behavior Research Methods, 38, 88-91.

Martín-Montañez, E., Barón-López, F. J., Rubio Lamia, L. O., Pavía Molina, J., Miranda Páez, J. y Santos Amaya, I. M. (2011). Consumo de alcohol, tabaco, cannabis y otras sustancias psicoactivas en estudiantes de la Universidad de Málaga. Trastornos Adictivos, 13, 160-166.

Martínez Delgado, J. M. (1996). Validación de los cuestionarios breves: AUDIT, CAGE y CBA para la detección precoz del síndrome de dependencia de alcohol en Atención Primaria. Tesis Doctoral no publicada. Cádiz: Universidad de Cádiz, España.

Meneses-Gaya, C., Waldo Zuardi, A., Regina Loureiro, S. R. y Crippa, J. A. (2009). Alcohol Use Disorders Identification Test (AUDIT): An Updated systematic review of psychometric properties. Psychology and Neuroscience, 2, 83-97.

Montaño, M. R., Morales Guarín, A., Gómez Millán, M. A., Vera Maldonado, A. y Gantiva Díaz, C. A. (2011). Consumo de alcohol en estudiantes universitarios. Psicogente, 14, 27-35.

Observatorio Español de la Droga y las Toxicomanías (2011). Encuesta Domiciliaria sobre Alcohol y Drogas en España (EDADES) 2011/12. Disponible en http:// www.pnsd.msc.es/Categoria2/observa/pdf/EDADES2011.pdf

Observatorio Español de la Droga y las Toxicomanías (2011). Informe 2011. Delegación del Gobierno para el Plan Nacional sobre Drogas. Disponible en http://www. pnsd.msc.es/Categoria2/observa/pdf/oed2011.pdf

Observatorio Europeo de las Drogas y las Toxicomanías (2011). El problema de la drogodependencia en Europa. Informe anual 2011. Disponible en http://www. emcdda.europa.eu/attachements.cfm/att_143743_ES_ EMCDDA_AR2011_ES.pdf

O’Malley, P. y Johnston, L. (2002). Epidemiology of alcohol and other drug use among American college students. Journal of Studies on Alcohol, 14, 23-39.

Pérula de Torres, L. A., Fernández-García, J. A., Arias-Vega, R., Muriel-Palomino, M., Márquez-Rebollo, E. y Ruiz Moral, R. (2005). Validación del cuestionario AUDIT para la identificación del consumo de riesgo y de los trastornos por el uso de alcohol en mujeres. Atención Primaria, 36, 499-506.

Reinert, D. F. y Allen, J. P. (2002). The Alcohol Use Disorders Identification Test (AUDIT): a review of recent 
research. Alcoholism: Clinical and Experimental Research, 26, 272-279.

Reinert, D. F. y Allen, J. P. (2007). The Alcohol Use Disorders Identification Test: an update of research findings. Alcoholism: Clinical and Experimental Research, 31, 185-199.

Rodríguez-Martos Dauer, A. (2007a). Primera conferencia de Prevención y Promoción de la salud en la práctica clínica en España. Prevención de los problemas derivados del consumo de alcohol. Ministerio de Sanidad y Consumo.

Rodríguez-Martos Dauer, A. (2007b) . ¿Por qué es tan difícil legislar sobre alcohol en España? Adicciones, 19, 325-332.

Rosón Hernández, B. (2008). Consumo de riesgo y perjudicial de alcohol. Prevalencia y métodos de detección en la práctica clínica. Galicia Clínica, 69, 29-44.

Rubio Valladolid, G., Bermejo Vicedo, J., Caballero Sánchez-Serrano, M. C. y Santo-Domingo Carrasco, J. (1998). Validación de la Prueba para la Identificación de Trastornos por Uso de Alcohol (AUDIT) en Atención Primaria. Revista Clínica Española, 198, 11-14.

Salazar Garza, M. L., Ávila Hernández, O. P., Pérez Castro, J. L. y Martínez Martínez, K. I. (2010). Detección temprana de jóvenes universitarios en riesgo por su consumo de alcohol. Investigación y Ciencia de la Universidad Autónoma de Aguascalientes México, 59, 40-46.

Saunders, J., Aasland, O., Babor, T., de la Fuente, J. y Grant, M. (1993). Development of the Alcohol Use Disorders Identification Test (AUDIT): WHO Collaborative project on early detection of persons with harmful alcohol consumption-II. Addiction, 88, 791-804.

Schmidt, A., Barry, K. y Fleming, M. (1995). A new screening test for the detection of problem drinkers: The Alcohol Use Disorders Identification Test (AUDIT). Southern Medical Journal, 88, 52-59.

Seguel Palma, F., Santander Manríquez, G. y Alexandre Barriga, O. (2013). Validez y confiabilidad del test de identificación de los trastornos debidos al consumo de alcohol (AUDIT) en estudiantes de una universidad chilena. Ciencia y Enfermería XIX, 1, 23-35.

Velicer, W. F. (1976). Determining the number of components from the matrix of partial correlations. Psychometrika, 41, 321-337.

Vinader-Caerols, C., Monleón, S. y Parra, A. (2014). Physiological and psychological effects of a high dose of alcohol in Young men and women. Adicciones, 26, 238-246.

Wicki, M., Kuntsche, E. y Gmel, G. (2010). Drinking at European universities? A review of students' alcohol use. Addictive Behaviors, 35, 913-924.

Zaldívar Basurto, F., López Ríos, F., García Montes, J. M. y Molina Moreno, A. (2011). Consumo autoinformado de alcohol y otras drogas en población universitaria espanola. Electronic Journal of Research in Educational Psychology, 9, 113-132. 